\title{
Being a "Dataologist": From Data to Networks to Personalized Healthcare
}

\author{
Nitesh Chawla
}

University of Notre Dame, USA

\begin{abstract}
In this talk, I will discuss our research in leveraging Big Data for the Common Good. I will provide an overview of different research initiatives in my research program in network and data science, including applications to grand societal challenges.

I will focus on our research initiative in patient-centered outcomes and population health management. Faced with unsustainable costs and enormous amounts of under-utilized data, health care needs more efficient practices, research, and tools to harness the full benefits of personal health and healthcare-related data. To that end, I will discuss a patient-centered population health management framework that can help us move from insufficient healthcare to abundant healthcare. I will also review our various pilots that are in play at various partner organizations.
\end{abstract}

